\title{
Importancia del entrenamiento técnico-táctico y de condición física en el proceso de formación de los judokas olímpicos españoles
}

\author{
Andrés ROBLES RODRÍGUEZ*1 (D), José ROBLES RODRÍGUEZ2 (D), Francisco Javier GIMÉNEZ \\ FUENTES-GUERRA2 (D) Manuel Tomás ABAD ROBLES $^{2}$ (†) \\ ${ }^{1}$ Centro Universitario San Isidoro, adscrito a la Universidad Pablo de Olavide, Sevilla (España) \\ 2 Universidad de Huelva (España)
}

I Congreso Nacional de Entrenadores de Judo, Murcia (España), 13-15 de julio, 2018

\section{Resumen}

Con el objetivo de conocer cómo se ha llevado a cabo el trabajo técnico-táctico y de condición física en el proceso de formación deportiva de los judokas españoles olímpicos, se aplicó una entrevista semi-estructurada a 20 judokas, concretamente a 12 mujeres (60\%) y 8 hombres (40\%). Los resultados muestran que los judokas olímpicos consideran que no han trabajo de un modo adecuado los aspectos tácticos durante su formación deportiva, además no hay unanimidad respecto a la edad en la que se debe profundizar en los aspectos técnicos. Por otra parte, la totalidad de los entrevistados dan mucha importancia al trabajo físico. La mayoría opinan que se debe trabajar de manera integrada, completándola con sesiones específicas. Consideran la fuerza como la capacidad física más importante en categorías de iniciación, mientras que en la élite son la resistencia y la fuerza.

Palabras clave: Judo; deportes de combate; élite; entrevista.
\end{abstract}

\section{Importance of technical-tactical and physical condition training in the training process of the Spanish} Olympic judokas

\begin{abstract}
In order to know how the technical-tactical and physical condition training has been carried out during the process of sports training of Spanish Olympic judokas, a semi-structured interview was conducted to 20 judokas - 12 women (60\%) and 8 men $(40 \%)$. The results show that the Olympic judokas consider that tactical aspects have not been training adequately during their sports career, besides there is no unanimity regarding the age in which the technical aspects should be worked intensively. On the other hand, all of the interviewees give great importance to physical training. Most of them believe that this training should be done in an integrated way, completing it with specific physical training sessions. They consider strength as the most important physical capacity at early age categories, while endurance and strength were considered the most important at the elite level.
\end{abstract}

Keywords: Judo; combat sports; elite; interview.

\section{Introducción}

En los últimos años numerosos estudios plantean el proceso de formación deportiva dependiendo de multitud de aspectos, y no sólo de la genética o de la cantidad de horas que dedica el deportista al entrenamiento (Lorenzo \& Calleja, 2010). En este mismo sentido, Ruiz y Salinero (2011) indican que el rendimiento del deportista hoy día no puede ser entendido sin una concepción integral del mismo, influyendo en el mismo diferentes aspectos. Entre ellos figuran aspectos técnicos, tácticos, físicos, psicológicos y contextuales (Robles, Robles, Giménez, \& Abad, 2016). En lo referente a los aspectos técnico-tácticos y físicos encontramos numerosas investigaciones que tratan de determinar su importancia, como las de Osipov et al. (2016) o Pion et al. (2015). El objetivo del presente estudio fue analizar la importancia del trabajo técnico-táctico y de condición física en el proceso de formación deportiva, desde el punto de vista de los judokas españoles que han participado en alguno de los Juegos Olímpicos celebrados en el siglo XXI (Sidney, Atenas, Pekín, Londres y Rio de Janeiro). 


\section{Método}

\subsection{Participantes}

La población objeto de estudio fueron los judokas españoles que han participado al menos una vez en unos juegos olímpicos en el Siglo XXI $(n=25)$. En la muestra del presente trabajo participaron 20 judokas (80\%): 12 mujeres (60\%) y 8 hombres (40\%) (Tabla 1). Se debe tener en cuenta que un mismo deportista puede haber participado en más de unos Juegos Olímpicos.

Tabla 1. Juegos Olímpicos, participantes y entrevistados.

\begin{tabular}{lcccc}
\hline Juegos Olímpicos & $\begin{array}{c}\text { Participantes } \\
\text { femeninos }\end{array}$ & $\begin{array}{c}\text { Participantes } \\
\text { masculinos }\end{array}$ & $\begin{array}{c}\mathbf{N}^{\mathbf{0}} \text { total de } \\
\text { participantes }\end{array}$ & $\begin{array}{c}\mathbf{N}^{\mathbf{0}} \text { total de entrevistados por } \\
\text { Juegos Olímpicos }\end{array}$ \\
\hline Sídney 2000 & 6 & 5 & 11 & 11 \\
Atenas 2004 & 4 & 6 & 10 & 10 \\
Pekín 2008 & 4 & 2 & 6 & 6 \\
Londres 2012 & 4 & 2 & 6 & 6 \\
Rio de Janeiro 2016 & 3 & 2 & 5 & 1 \\
\hline
\end{tabular}

\subsection{Instrumento}

Se utilizó una metodología cualitativa de investigación con el fin de conseguir una descripción profunda de los participantes en el estudio. El instrumento utilizado fue una entrevista semiestructurada diseñada y validada por Robles et al. (2016), constituida por 52 preguntas agrupadas en 6 dimensiones. El presente trabajo muestra los resultados de las siguientes dimensiones:

- Dimensión técnico-táctica: en la que se pretende analizar la importancia que los judokas dan a los contenidos técnico-tácticos, y también conocer su opinión en relación a cómo y cuándo lo trabajaron durante su proceso de formación.

- Dimensión condición física: se indaga sobre cómo se ha llevado a cabo el trabajo de condición física, quienes han sido los responsables del mismo, así como cuáles son las cualidades físicas que consideran más importantes para llegar a la élite.

\section{Resultados}

\subsection{Dimensión Técnico-Táctica}

En cuanto al trabajo técnico, el 95\% $(n=19)$ de los entrevistados lo consideraron muy importante. Encontramos, por un lado, aquellos que consideraban que se debe trabajar a partir de las categorías infantil o cadete $(45 \%, n=9) \mathrm{y}$, por otro lado, aquellos que opinaron que debe trabajarse desde los inicios de la práctica deportiva $(40 \%, n=8)$. El 80\% $(n=16)$ estaba satisfecho con el trabajo técnico durante su proceso de formación deportiva.

Respecto al trabajo táctico, el $100 \%$, lo consideraron muy importante durante la etapa de la élite deportiva; sin embargo, el 60\% $(n=12)$ opinaron que durante el proceso de formación le se dio poca importancia. En este sentido, ninguno de los entrevistados señaló que la táctica deba trabajarse antes de la categoría cadete (14-16 años). Sólo el 65\% $(n=13)$ consideraron que ha habían trabajado correctamente este aspecto.

\subsection{Dimensión Condición Física}

El $100 \%$ de los entrevistados consideran que el trabajo físico es de vital importancia, aunque no hay unanimidad respecto a cuándo debe comenzar a trabajarse dicho aspecto del entrenamiento. Por una parte, el $60 \%(n=12)$ de los judokas señalaron que el trabajo físico debía iniciarse a partir de cadetes (14-16 años), mientras que el $75 \%$ de los entrevistados $(n=15)$ opinaron que el trabajo físico debía ser ejecutado junto al trabajo técnico-táctico, es decir, de manera integrada. Por otro lado, el $55 \%(n=11)$ señalaron que también era importante realizar sesiones específicas de condición física. Llama la atención que la responsabilidad de trabajo físico 
del $90 \%(n=18)$ de los judokas olímpicos estuvo a cargo del propio entrenador de judo. En lo que respecta a las cualidades físicas fundamentales para llegar a ser un judoka de elite, los entrevistados consideraron que en la categoría inferiores es la fuerza la cualidad física primordial, aunque en la élite deportiva es la resistencia $(n=12)$, seguido de la fuerza $(n=9)$, y finalmente la velocidad $(n=5)$.

\section{Conclusiones}

Los entrevistados dan mucha importancia a los aspectos técnicos-tácticos, aunque valoran en mayor medida la táctica. La mitad consideran que la técnica hay que trabajarla al iniciar la práctica deportiva, la otra mitad afirma que en categorías más avanzadas como la infantil o cadete. La mayoría están satisfechos con el trabajo técnico realizado durante su formación deportiva. La totalidad de los entrevistados dan mucha importancia al trabajo físico. La mayoría opinan que se debe trabajar de manera integrada, aunque además hay que trabajar sesiones específicas. Consideran la fuerza como la cualidad física más importante en categorías de iniciación, mientras que en la élite son la resistencia y la fuerza.

\section{Referencias}

Lorenzo, A., \& Calleja, J. (2010). Factores condicionantes del desarrollo deportivo. Bizkaia: Diputación Foral de Bizkaia. Dirección General de Deportes.

Osipov, A., Kudryavtsev, M., Struchkov, V., Kuzmin, V., Bliznevsky, A., \& Plotnikova, I. (2016). Expert analysis of the competitive level of Young russian judo athletes who train for active attack fighting. Journal of Physical Education and Sport, 16(4), 1153-1158. doi: 10.7752/jpes.2016.04185

Pion, J., Segers, V., Fransen, J., Debuyck, G., Deprez, D., Haerens, L., \& Lenoir, M. (2015). Generic anthropometric and performance characteristics among elite adolescent boys in nine different sports. European Journal of Sport Science, 15(5), 357-366. doi: 10.1016/j.scispo.2014.08.065

Robles, A., Robles, J., Giménez, F. J., \& Abad, M. T. (2016). Validación de una entrevista para estudiar el proceso formativo de judokas de élite. Revista Internacional de Medicina y Ciencias de la Actividad Física y del Deporte, 16(64), 723-738. doi: 10.15366/rimcafd2016.64.007

Ruiz, G., \& Salinero, J. J. (2011). El entrenador de alto nivel en triatlón: entorno próximo y cualidades fundamentales para el rendimiento. International Journal of Sport Science, 23(7), 113-125. doi: 10.5232/ricyde2011.02304 